\title{
Albert Steuer Karfreitagsstimmung nach dem August-Erlebnis 1915 \\ Katholische Presse und kirchliche Publizistik im Ersten Weltkrieg
}

Als ein auch „globales Medienereignis“ und einen „Kampf um Begriffe und Bilder, um Sympathie und Anerkennung" beschreibt Michael Jeismann den Ersten Weltkrieg. ${ }^{1}$ Das erst 1871 begründete und damit historisch junge Deutsche Reich sah seit Beginn des 20. Jahrhunderts angesichts wachsender nationaler Spannungen in dem allseits erwarteten „Großen Krieg“ eine Chance, sich einen angemessenen Platz neben den etablierten $\mathrm{Großmächten} \mathrm{zu}$ erobern und $z \mathfrak{u}$ behaupten. ${ }^{2}$ Die seinerzeit existierenden rund 4000 Zeitungen in Deutschland kämpften diesen Krieg, durch den sie einerseits an Bedeutung gewannen, unter dem sie andererseits aber auch wirtschaftlich zu leiden hatten, engagiert mit.

Die konfessionell orientierte politische und kirchliche Presse ${ }^{3}$ schwamm überwiegend und vielfach bis zum bitteren Ende im Strom mit. Unter den bis zu 500 Tageszeitungen der katholischen Presse, meist kleine und kleinste Blätter mit einer Auflage unter 1000 Exemplare, waren während des Ersten Weltkrieges die „Kölnische Volkszeitung“ und die "Germania“ in Berlin zwar nicht die größten, sie gehörten aber mit ihren Redaktionen und deren Verbindungen $\mathrm{zu}$ den unterschiedlichen Strömungen der Katholiken-Partei sowie auf Grund ihres auch international erworbenen Ansehens zu den „Leit-Medien“ der ZentrumsPresse. ${ }^{4}$

1 Michael Jeißmann: „Propaganda“. In: Enzyklopädie Erster Weltkrieg (EEW), Hrsg.: Gerhard Hirschfeld und andere. Paderborn, München, Wien, Zürich 2003, S. 198209, hier S. 208.

2 Vgl. Wolfgang J. Mommsen: Der Erste Weltkrieg. Anfang vom Ende des bürgerlichen Zeitalters. Frankfurt 2004. Darin vor allem auch die Abhandlung "Die christlichen Kirchen im Ersten Weltkrieg“, S. 168-180. Sönke Neitzel: Weltmacht oder Untergang. Die Weltreichslehre im Zeitalter des Imperialismus. Paderborn, München, Wien, Zürich 2000.

3 Formal ist, auch wenn die Grenzen vielfach fließend waren, zu unterscheiden zwischen der "katholischen Presse" und der Kirchenpresse. Erstere umfasst die konfessionell orientierten politischen Tages- und Wochenzeitungen, die mehr oder minder der Deutschen Zentrumspartei als der politischen Organisation der Katholiken nahe standen, nicht aber einer kirchlichen Hierarchie unterstellt waren. Daneben waren,jm Laufe des 19. Jahrhunderts auch immer mehr kirchliche Blätter, etwa auf Diözesanebene, in Ordensgemeinschaften sowie Verbänden und wissenschaftlichen Kreisen erschienen.

4 Die „Kölnische Volkszeitung“ (Auflage 30 000) wurde 1860 von Joseph Bachem aus 
Wie auf protestantischer Seite auf Grund der „Ehe zwischen Thron und Altar" im wilhelminischen Deutschland, so waren auch auf katholischer Seite „Gott und Vaterland oftmals unauflöslich miteinander verwoben“. Statt auf Grund des doch gemeinsamen Glaubens nach Versöhnung zu suchen, bliesen sie, sich gegenseitig verstärkend, im Namen des jeweils eigenen „auserwählten Volkes" gegen die Feinde ins Horn zum gegenseitigen „Kreuzzug“. 5

"Der Karfreitag im Volkskriege!“ seufzte in merklichem Kontrast zu ihrer sonstigen Euphorie im Frühjahr 1915 die "Kölnische Volkszeitung“ und fragte gedämpft: „Ist nicht dieser gewaltige Krieg selber ein einziger

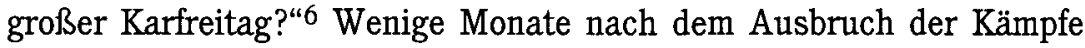
und trotz anhaltender Erfolge der Truppen der Mittelmächte gegen eine "Welt von Feinden" auf den Schlachtfeldern im Westen wie im Osten, brach die Stimmung angesichts der steigenden Zahl der Opfer dieses ersten technisierten und industrialisierten Materialkrieges ein. Die anfängliche Zuversicht des Deutschen Reiches und Österreich-Ungarns, den Ring der „Einkreisung“ durch die übrigen europäischen Großmächte rasch durchbrechen zu können, blieb alsbald in den blutigen Grabenkämpfen des Stellungskrieges stecken. Zwar wurde die bisherige Siegesgewissheit keineswegs aufgegeben, aber es waren zunehmend doch gedämpfte Töne - so etwa in der vorösterlichen Fastenzeit 1915 - zu lesen und immer häufiger auch Durchhalteparolen.

\section{Kriegsziel-Debatten und alldeutsche Phantasien}

Nach den als „Wort der Erlösung"7 begrüßten gegenseitigen Kriegserklärungen im August 1914 konfrontierte das Jahr 1915 die Kombattanten zunehmend mit der Frage nach dem Warum und Wofür. Zumal aus Sicht des Deutschen Reiches hatten sich die - in der ersten und keines-

Vorläuferorganen gegründet, war während des Ersten Weltkrieges das Hauptorgan des rheinisch-westfälischen Flügels der Zentrumspartei(„Mönchengladbach-Trierer“Richtung), verfügte über gute Kontakte zur Obersten Heeresleitung und erschien drei Mal täglich. Das formale Aus des Blattes kam am 29./30. April 1933, das endguiltige am 31. Mai 1941. Das hauptstädtische Zentrums-Organ, die „Germania Zeitung für das deutsche Volk" (Auflage zwischen 4000 und 8000/maximal 10000 ) entstand 1871 als unabhängige, der Leitung der Partei aber doch nahe stehende Publikation, was sich in Kommentierung und Meinungsäußerung niederschlug. Auch ihr Ende kam - unter den Nationalsozialisten - im Jahr 1938.

6 Kölnische Volkszeitung, Nr. 217 v. 2.4.1915.

7 Leuchtturm für Studierende, 7. Jg., Heft 24 v. 15.9.1914. 
wegs so verbreiteten Euphorie wie oft beschrieben - gehegten Hoffungen auf einen schnellen und erfolgreichen Feldzug „bis Weihnachten“ mit jedem Tag mehr als unrealistisch erwiesen. $\mathrm{Zu}$ jenen, die derartige Vorstellungen über einen kurzen Krieg mit vergleichsweise geringfügigen Opfern bereits sehr früh bezweifelten, gehörten auch einige katholische Bischöfe in Preußen. ${ }^{8}$

„Uns treibt nicht Eroberungslust", hatte Kaiser Wilhelm II. am 4. August 1914 als Devise ausgegeben, "uns beseelt der unbeugsame Wille, den Platz zu bewahren, auf den Gott uns gestellt hat, für uns und alle kommenden Geschlechter."9 Diese offizielle Losung vom Verteidigungskrieg war 1914/15 das der breiten Öffentlichkeit bekannte und von dieser mehr oder minder auch akzeptierte Kriegsziel, wie es im übrigen Reichskanzler Theobald von Bethmann Hollweg in immer neuen Varianten bekräftigte. „Wir mussten, wir konnten nicht anders!“, betonte zum Beispiel zum Neujahrstag 1915 der namentlich nicht genannte Leitartikler der „Kölnischen Volkszeitung“ und führte aus, Deutschland werde auch weil es an eine ,göttliche Weltregierung glaubt und seiner Vorsehung vertraut" - von der Vernunft geleitet, während hinter dem Angriffskrieg der Gegner Geld-, Machtgier und Streben nach Weltherrschaft stehe. ${ }^{10}$ Nicht wenigen Kräften im Reich, allen voran dem 1891 gegründeten und seit 1908 von Heinrich Claß geleiteten radikal-nationalistischen "Alldeutschen Verband" (ADV) und Kreisen der Wirtschaft, erschien die Bewahrung des Vorkriegsstatus, was im übrigen Kaiser und Regierung hinter den Kulissen de facto $\mathrm{zu}$ keiner Zeit beabsichtigten, alles andere als ein ausreichendes Kriegsziel.

Anders als in den gegnerischen Staaten verstärkte sich im Deutschen Reich trotz des von Kaiser Wilhelm II. und der Regierung ausgerufenen "Burgfriedens" und erster Zensurmaßnahmen bereits in den ersten Monaten eine lebhafte Debatte über die Kriegsziele. Erste Höhepunkte dieser Diskussionen, die an Großmachtsideen und Träume vom „Platz an der Sonne" anknüpften, die schon vor dem Krieg kursierten, fielen in das Jahr 1915. Ein Plädoyer für eine hegemoniale und expansionistische Politik des Deutschen Reiches kam auch aus der Deutschen Zentrumspartei. Ein maßgeblicher Mitautor war der Abgeordnete Matthias Erzber-

8 Hermann Josef Scheidgen: Deutsche Bischöfe im Ersten Weltkrieg. Die Mitglieder der Fuldaer Bischofskonferenz und ihre Ordinariate 1914-1918. Köln, Weimar, Wien 1991, S. 61, 70ff.

9 Ernst Heinen: Zentrumspresse und Kriegszieldiskussion. Köln 1961, S. 1. Vgl. Stenographische Berichte des Deutschen Reichstages, XIII. Legislaturperiode, Band 306-310, hier Bd. 306, S. 2.

10 Kölnische Volkszeitung, Nr. 2 v. 1.1.1915. 
ger, zu dieser Zeit noch Verfechter eines "Siegfriedens“.11 Derartige Ideen einer "deutschen Weltsendung“ kursierten selbst im linken und liberalen politischen Spektrum bereits in den Jahren vor"dem Kriegsausbruch. So konnte sich die Regierung von Reichskanzler von Bethmann Hollweg, auch wenn sie ihre Ziele aus taktischen Gründen nicht publik machte, doch eines breiten Rückhaltes in den Reihen der Intellektuellen sowie in den elitären Schichten sicher sein.

$\mathrm{Zu}$ jenen Blättern der katholischen Presse, die offensiv eine Hegemonialstellung des Deutschen Reiches propagierten und die Kriegszieltaktik des Reichskanzlers von Bethmann Hollweg als „Flaumacherei“ angriffen, gehörte - soweit Zensur und Selbstzensur es erlaubten - insbesondere die „Kölnische Volkszeitung“. Aber auch eher unpolitische und erbauliche kirchliche Publikationen sahen, wie etwa im Juli 1915 die im Erzbistum Köln erscheinende „Katholische Kirchenzeitung“, die bereits tot gesagte „alte, deutsche, germanische Kraft" wieder aufleben und priesen diese in oft pseudoreligiöser Begeisterung als erste „Frucht und Segnung" des um die Existenz des Reiches geführten Krieges. An der eigenen Stärke und Größe, an diesem „neu erwachten ,Deutschtum ' gilt es festzuhalten". Ebenso seien in der langen Friedenszeit vernachlässigte Tugenden und der Glauben wieder erweckt worden. ${ }^{12}$ Einen ähnlichen Gedanken präsentierte zu Ostern die „Allgemeine Rundschau“ in München: „Man kann nicht einmal sagen, dass der Krieg erfolgreicher ist für die Religion. Ja es kann sogar sein, dass der Krieg eine Friedenszeit für die Religion, der kommende Friede aber eine Kriegszeit wird. Wie viel Frieden bringt die Religion dem Menschen in den Wirren und Nöten des Krieges, und wie viel innere und äußere Kämpfe bringt sie ihm in Friedenszeiten." ${ }^{13}$ Vom Kriegsausgang, der für Deutschland natürlich den Sieg bedeuten werde, erhoffte wiederum die Kirchenzeitung in Köln unter

11 Matthias Erzberger (1875-1921), Lehrer und Redakteur der Zentrumszeitung „Deutsches Volksblatt“, saß seit 1903 für die Deutsche Zentrumspartei im Berliner Reichstag. Im Verlauf des Ersten Weltkrieges wandelte er seine Einstellung hinsichtlich expansionistischer Ziele und gehörte $1917 \mathrm{zu}$ den maßgeblichen Initiatoren jener Reichstagsresolution, die sich, wie die von Papst Benedikt XV. zum 1. August 1917 veröffentlichte Friedenskundgebung, für einen Verständigungsfrieden einsetzte. Als Staatssekretär unterzeichnete er im November 1918 in Compiègne die deutsche Kapitulation und setzte sich für die Annahme des Versailler Vertrages ein. In der Weimarer Republik wurde er Finanzminister. Am 25. August 1921 fiel er einem Attentat zum Opfer.

12 Katholische Kirchenzeitung Köln, Nr. 23 v. 6.6.1915. Hervorhebungen im Blatt. Unter gleichem Obertitel erschien diese Zeitung in mehreren Pfarreien des Erzbistum Köln, u.a. in St. Maternus und St. Ursula.

13 Allgemeine Rundschau, XII. Jg., Nr. 14 v. 3.4.1915. 
anderem die Wiederherstellung der alten Ordnung, des „patriarchalischen Verhältnisses zwischen Herrn und Arbeiter", wobei es allerdings mit der "Ausbeutungspolitik und Aussaugungsmethode" seitens der Großindustrie ein Ende habe müsse. Und nicht zuletzt gelte es, den unter dem Burgfrieden erreichten Zustand der Einigkeit zwischen Parteien und Konfessionen in die künftige Friedenszeit hinüberzuretten. ${ }^{14}$

Häufig findet sich der Vergleich zwischen der zurückliegenden Friedenszeit, in der Wertebewusstsein und Religion einen bedenklichen Niedergang erlebt hätten, und der Gegenwart, in der sich der Krieg als „Glaubenskünder" erweise, wie ihn Otto Zimmermann SJ in der JesuitenZeitschrift „Stimmen aus Maria Laach“ anstellte. Die Deutschen seien wieder, begrüßte er, vom Bewusstsein für die Bedeutung der Religion erfasst und das "ganze Land zum Gotteshaus" geworden. Ebenso erweise sich der Krieg als „große Mission“ und „erschütternde Exerzitien“. Die oft verbreitete Vereinnahmung, dass Gott mit den Armeen Deutschlands und Österreichs kämpfe, bejahte Zimmermann zwar auch, ergänzte sie aber - zu dieser Zeit durchaus bemerkenswert - um den Hinweis: „Gott steht über den Kämpfen, soweit sie nur Nationen vertreten; Gott steht beim Recht [...]. In Gott werden dann, wenn die Kämpfe vorüber sind, die Völker wieder zum Frieden finden."15

Beachtenswert erscheint ferner die philosophisch-theologische Abhandlung über „Die sittliche Berechtigung und Bedeutung des Krieges“ aus der Feder von August Messer im März-Heft der Kulturzeitschrift „Hochland“. Bei aller generellen Zustimmung zur deutschen Position ist dieser Artikel doch eine differenziertere Wortmeldung zur Thematik wenn auch eine der wenigen. In dem allerdings schon vom Sprachduktus her nicht an eine breite Leserschaft gerichteten Beitrag wandte sich Messer gleichermaßen gegen Konzepte, die den Krieg in einer utilitaristischen Ethik ablehnen, weil er dem Glück des Menschen entgegenstehe, oder die im Krieg einen Eigenwert sehen. Eingrenzend hielt Messer den Krieg vom christlichen Standpunkt her für gerechtfertigt, wenn er „als das einzig mögliche Mittel zur Erhaltung eines sittlichen Eigenwertes ausgewiesen werden “ könne, wie ihn etwa Erhalt und Förderung „nationaler Kulturstaaten" darstellten. Nur ein aufgezwungener und zur Verteidigung geführter Krieg sei ein gerechter, und das kriegführende Volk sei verpflichtet, diesen „menschlich, d.i. sittlich, zu führen“. Ziel müsse ferner sein, einen dauerhaften Frieden zu sichern. Daher sollten auch die

14 Katholische Kirehenzeitung Köln, Nr. 23 v. 6.6.1915.

15 Otto Zimmermann: Glaubenskünder Krieg. In: Stimmen aus Maria Laach, 88. Jg. 1915, Heft 5 (Mai). Die Jesuiten-Zeitschrift benannte sich erst zur Jahreswende 1916/17 in "Stimmen der Zeit“ um. 
Staaten - wie auf menschlich-individueller Ebene üblich - ein̨en gewaltsamen Streit durch gegenseitige Zugeständnisse schlichten. ${ }^{16}$ Ein $\mathrm{Ge}$ danke, um den in den folgenden Kriegsjahren publizistisch noch heftig gerungen wurde.

Ohne im Krieg einen letzten Wert zu sehen, hob in der Paderborner Zeitschrift "Theologie und Glaube" der Theologe Franz Sawicki grundsätzlich als dessen positive Begleiterscheinungen vor, dass die militärische Auseinandersetzung das Bewusstsein für die Zugehörigkeit $\mathrm{zu}$ einem Staat fördere und politische, wirtschaftliche sowie religiöse Gegensätze überbrücke. Er sah die Weltgeschichte unter der „Idee der Gerechtigkeit" stehen, gab aber zu bedenken, dass damit nicht unbedingt vorübergehende Erfolge des Bösen und ein zeitweiliges Unterliegen des Guten ausgeschlossen seien. Die eigentliche Frage sah Sawicki darin, ob Gottes Gerechtigkeit geschmälert würde, wenn er nicht im Diesseits für einen vollkommenen Ausgleich sorge. Dass Gott „immer mit der gerechten Sache ist“, führte er aus, schließe ein, dass er um seiner Vorsehung willen auch ein Volk trotz schwerer Schuld zu Macht und Wohlstand gelangen, ein anderes aber aus pädagogischen Gründen leiden lassen könne. Angesichts der historischen und mit der Vernunft erkennbaren Hinweise auf das gerechte Walten Gottes, schloss Sawicki gleichwohl zuversichtlich, könne Deutschland aus dem Bewusstsein, für eine gerechte Sache zu kämpfen, „ein gewisses Vertrauen auf deren guten Ausgang schöpfen“, und dies, obwohl Gott die ganze Geschichte seines Volkes kenne und die der Deutschen nicht frei von Schuld sei. ${ }^{17}$ Von solch einer auch nur ansatzhaften historischen Relativierung hielt wiederum Wilhelm Irmsee nichts, der in seinem Beitrag im „Leuchtturm für Studierende" zum ersten Jahrestag des Kriegsausbruchs im August 1915 die Deutschen gar zu „Königen der Erde“ kürte. Ein Erfolg sei sicher, gab es für ihn nicht die geringsten Zweifel, handelten doch politische und militärische Führung im Sinne des „Allerhöchstkommandierenden“. Mit dem Sieg verknüpfte Irmsee die Hoffnung auf eine Re-Christianisierung des Landes als notwendiges Fundament für dessen künftige Weltstellung - „für Gott und darum für das Vaterland“. 18

Mitte Oktober 1915 beriet erstmals der Reichsausschuss des Zentrums in Frankfurt/Main über die Haltung der Katholiken-Partei zu den Kriegszielen. Die Berliner „Centrums-Parlaments-Correspondenz“ (CPC)

16 Hochland, 12. Jg., Heft 6 (März) 1915, S. 641-659, hier S. 647 und 659. Hervorhebung im Blatt.

17 Franz Sawicki: Weltgeschichte als Weltgericht. In: Theologie und Glaube, März 1915, S. 183-191.

18 Leuchtturm für Studierende, 8. Jg., Heft 21-23 v. 1.8.1915, S. 481. 
lieferte dazu den Zeitungen die zusammenfassende Information, die formal auf der offiziellen Regierungslinie lag, in einzelnen Formulierungen jedoch durchaus expansionistisch interpretierbar war: „Die Weltaufgabe, die der Tüchtigkeit und dem Ziele des deutschen Volkes gesetzt worden ist, fordert die volle Bewegungsfreiheit seiner schaffenden Kräfte in der Heimat, auf dem freien Meer und über See. Die äußere Bedingung für eine gedeihliche Entwicklung des deutschen Volkes ist, wie die Erfahrungen des Weltkrieges klar gezeigt haben, eine erhöhte Sicherheit gegen die militärischen und wirtschaftlichen Vernichtungspläne der Feinde. Die furchtbaren Opfer, die der Krieg unserem Volke auferlegt, rufen nach einem verstärkten Schutze unseres Landes im Osten und Westen, der es den Feinden verleidet, uns wieder zu überfallen und der die volkswirtschaftliche Versorgung unserer wachsenden Bevölkerung dauernd gewährleistet. Zu dieser erhöhten Sicherung unseres Reiches muß eine solche unserer verbündeten Staaten treten. "19 Während sich im Verlauf des Jahres 1915 das rechte Parteienspektrum hinter der Reichsleitung schloss, begannen auf der anderen Seite die Sozialdemokraten ihre Zustimmung zum Krieg zunehmend zu hinterfragen. Am 9. Dezember schließlich forderte die SPD-Reichstagsfraktion in einer Interpellation von der Regierung Auskunft über ihre Kriegszielvorstellungen, wobei die Sozialdemokratie in ihrer Begründung erkennen ließ, dass es ihr um einen Frieden ohne Annexionen und die Sicherung des Status der Vorkriegszeit gehe. ${ }^{20}$

In seiner Antwort legte sich Reichskanzler von Bethmann Hollweg nicht klar fest, deutete allerdings Bereitschaft zu einem gemäßigten Annexionsfrieden an, woraus die Berliner „Germania“ und viele weitere Zentrums-Blätter eine nochmalige Bekräftigung des Verteidigungsfriedens herauslasen, der von allen Deutschen in Einigkeit getragen werde. ${ }^{21}$ Anders interpretierte die „Kölnische Volkszeitung“ die Kanzler-Rede dahingehend, dass die Regierung zwar immer noch „grob umrissen“, aber doch „,in der Tat ein festes Programm" dargeboten habe, das hinsichtlich der Kriegsziele deutlichere Garantien beinhalte. ${ }^{22}$ Dies bestärkte das Blatt, am folgenden Tag in einem Kommentar nur mühsam verklausuliert zu betonen: „Sicherheit ist es, die wir verlangen, und die wir gestützt auf Faustpfänder, die wir militärisch fest in der Hand halten, auch vom strengsten realpolitischen Standpunkt zu verlangen und durchzusetzen

19 Ernst Heinen: a.a.0., S.16.

20 Ernst Heinen: a.a.0., S. 17. Vgl. Stenographische Berichte des Deutschen Reichstages, Bd. 307, S. $430 \mathrm{ff}$.

21 Germania, Nr. 596 v. 10.12.1915.

22 Kölnische Volkszeitung, Nr. 1008 v. 9.12.1915. 
berechtigt sind. [...] Die Garantien, die wir fordern, sind in der Tat gar nicht zu vergleichen mit den Phantastereien unserer Feinde. Aber so weit geht freilich unsere Bescheidenheit nicht, dass wir bei Fortsetzung des Krieges auch noch bewogen werden könnten, auf die notwendigen Garantien für unsere Sicherung zu verzichten." 23

\section{Idealisierung des Kaisers Wilhelm II.}

An den viel zitierten Ausruf Kaiser Wilhelm II. ${ }^{24}$ Anfang August 1914, er kenne nur noch Deutsche, aber keine Partei- oder Konfessionsgrenzen mehr, knüpften viele katholische Intellektuelle und insbesondere die Politiker der Deutschen Zentrumspartei die konkrete Hoffnung, durch den Erweis nationaler Zuverlässigkeit, patriotischer Begeisterung und Opferbereitschaft im Krieg endgültig im protestantisch dominierten Deutschen Reich ihren gesellschaftlichen „Untermieterstatus“ überwinden und die immer noch spürbaren Nachwehen der Kulturkampfzeiten unter Otto von Bismarck beenden zu können. ${ }^{25}$ In diesem nationalen Streben war - vor allem im katholischen Bürgertum - bereits zu Beginn des 20. Jahrhunderts eine gewisse Aussöhnung mit dem deutschen Kaiserreich auf der Basis der kleindeutschen Lösung erfolgt. Und hier liegt, wie der Blick in die katholische Presse und die Kirchenpresse dieser Jahre zeigt, auch eine Basis für eine Kriegsbegeisterung der Katholiken, die jener der Protestanten kaum nachstand. Das „August-Erlebnis“ 1914 hat diese nationale Emphase verstärkt und „die inneren Spannungen im Katholizismus für einige Zeit ziemlich verdeckt“26; unterschwellig aber wirkten diese weiter.

Mit Kriegsausbruch, und erst recht angesichts ausländischer Propaganda, die vor der Person des deutschen Kaisers nicht Halt machte, trat auch für die katholische und die kirchliche Presse in Deutschland die protestantische Konfession Wilhelm II. zurïck hinter einer als allgemein

23 Kölnische Volkszeitung, Nr. 1010 v.10.12.1915.

24 Wilhelm II. (1859-1941) bestieg im „Drei-Kaiser-Jahr“ 1888 den Thron. Bei Kriegsende 1918 zur Abdankung gedrängt, ging er ins niederländische Exil, wo er am 4. Juni 1941 starb.

25 Wilfried Loth: Katholiken im Kaiserreich. Düsseldorf 1984. Rudolf Morsey: Die deutschen Katholiken und der Nationalstaat zwischen Kulturkampf und Erstem Weltkrieg. In ders.: Von Windthorst bis Adenauer. Paderborn, München, Wien, Zürich 1997, S. 158-186.

26 Wilfried Loth: Politischer Katholizismus in Deutschland: Entstehung, Antriebskräfte, Verfall. In: Franz-Xaver Kaufmann/Arnold Zingerle: Vatikanum II und Modernisierung. Paderborn, München, Wien, Zürich 1996, S. 35-52, hier S. 47. 
für vorbildlich gehaltenen und entsprechend dargestellten christlichen Glaubenshaltung. Exemplarisch zeigte dies etwa der Hymnus „Dem Kaiser!“, den Wilhelm Irmsee ein halbes Jahr nach Kriegsausbruch in der Zeitschrift „Leuchtturm für Studierende“ veröffentlichte. ${ }^{27}$ Als „Herrschernatur", die alle anderen europäischen Monarchen weit überrage, und als besonnenen "Charakterkopf" verteidigte der Katholik Irmsee seinen protestantischen Kaiser gegen äußere Angriffe. Für das Emporstreben Deutschlands habe Wilhelm II. als „Friedenskaiser“ in den zurückliegenden Jahren unermüdlich und erfolgreich gearbeitet und angesichts des wachsenden Neides der übrigen Staaten gegen das aufsteigende Deutsche Reich alles unternommen, einen Krieg zu verhindern.

Derartige Bewunderung für Wilhelm II. bestärkte nicht nur die Blätter protestantischer, sondern auch die katholischer Provenienz in Furcht vor drohenden republikanischen Umwandlungen, die im übrigen durch den Krieg zunächst ebenfalls hinausgezögert wurden, im Festhalten am „Gottesgnadentum" der Monarchie, sei doch insbesondere die religiöse Rückkoppelung weltlicher Macht in den „Wogen der Demokratie“ ein „tief konservativer glücklicher Gedanke“. ${ }^{28}$ Aus der Überhöhung der Person des Kaisers erwuchs die Hoffnung auf eine Vollendung der inneren Einheit des Deutschen Reiches und eine Überwindung der doch immer wieder zu Tage tretenden konfessionellen Spannungen zwischen den 62 Prozent Protestanten und den 36,5 Prozent Katholiken. In beiden großen Konfessionen, vor allem aber im staatskirchlich organisierten Protestantismus, waren die vornehmlichen Tendenzen noch von gegenseitiger Abgrenzung bestimmt. Der Krieg könne und werde sich als Zäsur, so der häufig formulierte Wunsch in der katholischen Presse und in kirchlichen Blättern, zwischen dem „alten" und dem "neuen" Deutschland erweisen. Dabei gehe es nicht, so etwa im Frühjahr die Münchner „Allgemeine Rundschau“, um Gleichmacherei und Verschmelzung, sondern um friedliche und gleichberechtigte Koexistenz der Konfessionen. ${ }^{29}$

27 Wilhelm Irmsee; „Dem Kaiser!“ In: Leuchtturm für Studierende, 8. Jg., Heft 8 v. 15.1.1915, S. 169-171.

28 Wilhelm Irmsee: a.a.0. S. 170.

29 Allgemeine Rundschau, Nr. 15 v. 10.4.1915. 


\section{Der deutsch-belgische Konflikt um Kardinal-Primas Mẹcier}

Aufsehen galt zur Jahreswende 1914/15 der durch den" belgischen Kardinal-Primas Desiré Mercier ${ }^{30}$ ausgelösten Krise. Der Erzbischof von Mechelen verband seit dem völkerrechtswidrigen Einmarsch Deutschlands in das neutrale Belgien im August 1914 immer wieder mit ermutigenden pastoralen Worten an seine Landsleute überaus deutliche Kritik an den Besatzungstruppen. In der Überzeugung, dass das Eintreten für das Vaterland stets auch ein bewusstes oder unbewusstes Glaubensbekenntnis sei, bejahte der belgische Primas einen Widerstand mit allen Kräften gegen die Deutschen, bis das Königreich wiederhergestellt sei. „Diese Kundgebung des Herrn Kardinals“, hielt sich die „Kölnische Volkszeitung" am 11. Januar 1915 im Kommentar über Merciers Hirtenbrief zum Jahreswechsel vergleichsweise zurück, „tritt aus dem Rahmen eines rein kirchlichen Erlasses" hinaus. ${ }^{31}$ Was dem Zentrums-Blatt aus dem Mund deutscher Bischöfe wohl als willkommene und selbstverständliche Verteidigung des Vaterlandes und Rückenstärkung für die kämpfenden Truppen erschienen wäre, kreidete es dem belgischen Primas als „Ausfluß patriotischer Überreiztheit“ an.

Bereits zu Weihnachten 1914 hatte Kardinal Mercier in seinem Hirtenbrief mit dem durchaus politischen Titel "Patriotisme et Endurance“ (Patriotismus und Widerstandskraft), in welchem er den Bruch der Neutralität Belgiens durch das Deutsche Reich scharf kritisierte, Aufsehen und politische Beunruhigung bewirkt. Seit König Albert I. ${ }^{32}$ und das Parlament vor den einrückenden deutschen Truppen die Hauptstadt Brüssel verlassen hatten, stand der Primas als höchste verbliebene Autorität im okkupierten Teil seines Landes dem deutschen Generalgouverneur Moritz Freiherr von Bissing gegenüber und war aus nationalpatriotischen wie auch aus kirchenpolitischen Motiven heraus gewillt, diese Position so weit wie möglich zu nutzen. Auf Grund dieses Engagements, dass neben politischen auch zunehmend innerkirchliche Beden-

30 Desiré-Félicien-Francois-Joseph Mercier (1815-1926) wurde 1906 von Papst Pius X. zum Erzbischof von Mechelen, traditionell auch Sitz des belgischen Primas, ernannt und 1907 zum Kardinal erhoben. Vgl. Roger Aubert: Les deux premiers grands conflicts du Cardinal Mercier avec les autorités allemandes d'occupation. Louvain-la-Neuve 1998. Angesicht anti-katholischer politischer Tendenzen im katholischen Belgien der Vorkriegszeit sah Mercier im Krieggeschehen eine Möglichkeit, durch eindeutig patriotische Positionen die Stellung von Kirche und Katholizismus im Königreich wieder zu festigen.

31 Kölnische Volkszeitung, Nr. 30 v. 11.1.1915. Inhaltlich gab sie den Hirtenbrief nicht detailliert wieder.

32 Albert I. (1875-1934) hatte 1909 den belgischen Königsthron bestiegen. 
ken bewirkte, ersuchte Anfang 1915 der Vorsitzende der Fuldaer Bischofskonferenz, der Kölner Kardinal Felix von Hartmann, den niederländischen Kardinal Willem Marinus van Roussum ${ }^{33}$, beim Heiligen Stuhl darauf hinzuwirken, dass Mercier aus der "Schusslinie“ genommen und an die Kurie versetzt werde. Gegen dieses Ansinnen intervenierte gleichwohl der preußische Gesandte beim Heiligen Stuhl, Otto von Mühlberg, weil Berlin dem belgischen Kardinal nicht zu einer irgendwie gearteten „Märtyrerrolle" verhelfen und jedes unkalkulierbare Aufsehen um dessen Person vermeiden wollte. Mühlberg signalisierte allerdings die klare Erwartung, dass Papst und Kurie mäßigend auf Mercier einwirken sollten. ${ }^{34}$ Im Sommer 1915 griffen die beiden führenden ZentrumsBlätter den weiter schwelenden Konflikt erneut auf.

Das Gebaren des belgischen Kardinals, meinte die "Germania“ Anfang Juli, demonstriere exemplarisch, mit welchem Eifer die Feinde bestrebt seien, „unserem Vaterland im allgemeinen und unserer Kriegsführung im besonderen die schlimmsten kirchenfeindlichen Tendenzen anzudichten". ${ }^{35}$ Das Ausland belebe propagandistisch die Kontroversen des Kulturkampfes wieder, obwohl diese „Verirrung bismarckscher Politik“ längst beigelegt sei. In den gegnerischen Staaten jedoch werde alles, was die „Katholikenfeindschaft des Deutschtums“ zu bestätigen scheine, gläubig aufgenommen und mit „patriotischem Übereifer“" weitergegeben. ${ }^{36}$ Dankbar zitierte die "Germania“ die vom Papst in einem Interview mit der französischen Zeitung "Liberté" gemachte Klarstellung, mit der Benedikt XV. den deutschen Katholiken diplomatisch und mäßigend bescheinigte, dass sie ihr „so unrecht und übel verleumdetes Vaterland besser kennen" und wüssten, dass Kardinal Mercier jede Kirchenfeindlichkeit nach innen wie nach außen fremd sei. Damit sollte und müsste, hoffte die Berliner Zeitung auf einen Schlussstrich, die "ganze ,Affaire", die Deutschlands Feinde so übel aufgebauscht“ hätten, „nun endgültig

33 Kardinal Willem Marinus van Roussum (1854-1932) war seinerzeit Präsident der Päpstlichen Bibelkommission an der römischen Kurie.

34 Ilse Meseberg-Haubold: Der Widerstand Kardinal Merciers gegen die deutsche Besatzung Belgiens 1914. Frankfurt/Main, Bern 1982, S. 66ff. Kardinal von Hartmann hatte bereits in einem Schreiben vom 6.1.1915 Generalgouverneur von Bissing sein Bedauern über das Agieren des Erzbischofs von Mechelen bekundet, und der Paderborner Bischof Karl Joseph Schulte äußerte, Merciers WeihnachtsHirtenbrief sei schlimmer als erwartet ausgefallen; mehr Besonnenheit wäre wünschenswert. Vgl. Hermann-Josef Scheidgen: Deutsche Bischöfe im Ersten Weltkrieg, S. 302.

35 Germania, Nr. 308 v. 8.7.1915. Kölnische Volkszeitung, Nr. 638 v. $7.8 .1915 /$ Nr. 706 v. 30.8 .1915 .

36 Germania, Nr. 308 v. 8.7.1915. 
als begraben“ und "restlos geklärt" betrachtet werden. ${ }^{37}$. Auch die „Kölnische Volkszeitung“ übte in dieser Angelegenheit vergleichsweise Zurückhaltung und versicherte, sollte das Deutsche Reich nach dem Krieg einen bestimmenden Einfluss auf das Geschick des mehrheitlich katholischen Königreichs ausüben, „so muß und wird als einer der ersten Grundsätze die bisherige Freiheit der katholischen Kirche in Belgien gelten ". 3 8 Solange im übrigen der dortige Katholizismus nicht von bestimmten Kräften national-politisch eingefärbt und gegen Deutschland instrumentalisiert werde, falle es nicht schwer, diese Rechte anzuerkennen.

Als Ende Oktober 1915 der Apostolische Nuntius in Belgien, Erzbischof Giovanni Tacci, bei den deutschen Behörden für Kardinal Mercier eine Ausreisegenehmigung nach Rom - offiziell zu einer Reorganisation der Studienkongregation - beantragte, spitzte sich der Konflikt wieder zu. Umgehend keimten Gerüchte, der Erzbischof werde auf Ersuchen Deutschlands nach Rom zitiert und seine Heimkehr sei unwahrscheinlich. Noch war Merciers Hirtenbrief vom 29. September 1915 in Erinnerung, in dem der Primas deutliche Parallelen zwischen Luzifer und seinem Gefolge sowie dem deutschen Kaiser und seinen Truppen gezogen hatte. Daraufhin hatte Reichskanzler von Bethmann Hollweg beim Vorsitzenden der Fuldaer Bischofskonferenz, Kardinal von Hartmann, interveniert und diesen gebeten, er solle - im Interesse Deutschlands und der katholischen Kirche - Papst Benedikt XV. persönlich über die Situation informieren. Der Kölner Kardinal unterbreitete Ende November in Rom mehrere Vorschläge, Mercier zu größerer Zurückhaltung zu veranlassen und damit dessen drohende Verhaftung zu vermeiden. Begleitet von überaus versöhnlichen Tönen des deutschen Episkopats-Vorsitzenden reiste der belgische Primas dann im Januar 1916 nach Rom. Die Angelegenheit war jedoch keineswegs beigelegt, warb Mercier doch auch hier klar für seinen und für den Standpunkt seines Landes. ${ }^{39}$

\section{Die französisch-deutsche Katholikenfehde}

Hielten sich Deutschlands Katholische Presse und die kirchlichen Blätter angesichts der allseits tangierten Personen in der Behandlung der „Affäre Mercier" noch zurück, so prallten in der zeitgleich ausgetragenen Fehde zwischen französischen und deutschen Katholiken die Positionen sehr viel direkter und feindseliger aufeinander. Gerade dieser

37 Germania, Nr. 308 v. 8.7.1915.

38 Kölnische Volkszeitung, Nr. 638 v. 7.8.1915. Hervorhebung im Blatt.

$39 \mathrm{Vgl}$. Hermann-Josef Scheidgen: a.a.0., S. 618f. 
Theologen- und Medienstreit, letztlich nur für einen kleinen Kreis nachvollziehbar und zu beurteilen, veranschaulicht, wie unter den Einwirkungen des Ersten Weltkrieges patriotisch-nationale Gefühle ins Übermaß gesteigert und die Religion instrumentalisiert wurden, um die jeweils eigene Position zu rechtfertigen, und wie nicht nur das gemeinsame christliche, sondern innerkatholisch auch das weltkirchliche Band zerschnitten wurde. Zur Person des Papstes und seinem Friedensengagement bekannten sich verbal alle Staaten und alle Christen. Und wenn damit auch das katholische Kirchenoberhaupt und der Heilige Stuhl in den Jahren des Ersten Weltkrieges international an Ansehen gewannen, Zustimmung gab es meist nur, wenn sich Äußerungen und Aktionen des Papstes für eine Seite vereinnahmen ließen oder dortigen Interessen zumindest nicht zuwiderliefen.

Deutschland habe den "Großen Krieg“ gewollt und verschuldet, schleuderten französische katholische Intellektuelle - darunter auch elf Bischöfe - im Frühjahr 1915 als zentrale Anklage in der Schrift „La Guerre allemande et le Catholicisme" (Der deutsche Krieg und der Katholizismus) und der bebilderten Dokumentation „Album $\mathrm{N}^{\circ} 1 \mathrm{La}$ Guerre allemande et le Catholicisme" dem deutschen Staat und damit auch den dortigen Katholiken entgegen. ${ }^{40}$ Hier lösten die als "Kampfschriften“ aufgefassten französischen Publikationen ein erbostes Echo aus, wurden die Deutschen doch nicht nur als Feinde des Katholizismus, sondern des Christentums überhaupt verunglimpft. Von der Intention und dem Sprachduktus her zwar vorrangig eine Kontroverse zwischen Intellektuellen, verhalf die Presse in beiden Ländern dem Streit zu einer breiteren Aufmerksamkeit, vor allem im Zeitraum zwischen Juni bis September 1915 , letztlich aber über dieses Jahr hinaus.

Abgesehen von einem eher vordergründigen deutschen Medienecho ließ die fundiertere, von nationaler Polemik allerdings ebenfalls nicht freie Antwort katholischer Gebildeter im Deutschen Reich nicht lange auf sich warten. Zunächst erschien der Sammelband „Der deutsche Krieg und der Katholizismus. Deutsche Abwehr französischer Angriffe" unter maßgeblicher Beteiligung des katholischen Paderborner Theologen Arnold Joseph Rosenberg. Anders als auf französischer Seite gehörte zu den Initiatoren allerdings - mit Ausnahme des Generalvikars Franz

40 Alfred Baudrillart (Hrsg.): La Guerre Allemande et le Catholicisme. Ouvrage sous le haut Patronage du Comité Catholique de Propaganda Francaise a l'Etranger. Paris 1915. (Der deutsche Krieg und der Katholizismus). Ders.(Hrsg.): Album $\mathrm{N}^{\circ} 1 \mathrm{La}$ Guerre Allemande et le Catholicisme. Paris 1915. Zu dem Buch verfasste der Pariser Erzbischof, Kardinal Léon-Adolphe Amette, ein Geleitwort. 
Scharmer von Kulm - kein Mitglied der Kirchenhierarchie in Preußen. ${ }^{41}$ Erst an der zweiten Abwehrpublikation „Deutsche Kultur, Katholizismus und Welt", herausgegeben von dem katholischen Freibürger Kirchenhistoriker Georg Pfeilschifter ${ }^{42}$, war dann mit dem damaligen Speyerer Bischof Michael Faulhaber ${ }^{43}$, in Personalunion katholischer Militärbischof im Königreich Bayern, ein Mitglied der Freisinger Bischofskonferenz mit einem eigenen Beitrag beteiligt.

Das „Gesamtbild ist falsch“, resümierte Rosenberg im Mai 1915 in einer Entgegnung in der Paderborner Zeitschrift "Theologie und Glaube“ zu den bis dahin nur in französischer Sprache vorliegenden "La Guerre“Publikationen. ${ }^{44}$ Zwar zeigte er ein gewisses Verständnis für die im Kriegsgebiet Frankreich vertretenen Positionen, warf insgesamt jedoch den Autoren „entsetzliche Einseitigkeit" vor. Entschieden trat Rosenberg dem Ansinnen entgegen, die Äußerungen Papst Benedikt XV. nur in einem französischen Sinne auszudeuten. Die „Kölnische Volkszeitung“ befasste sich erstmals im Juni ausführlich mit den „überaus schmerzlichen" französischen Schriften; diese "dienen nicht der Versöhnung und dem Frieden, und sie dienen auch nicht dem Katholizismus". Vielmehr werde durch eine „gekünstelte Auslegung“ die eigene „katholische“ Bedeutung "national“ gefärbt und "nationale Leidenschaft in das Feuer sengenden und verzehrenden Hasses" geblasen. ${ }^{45}$ Zustimmung in der Katholischen Presse fand im Juni das gemeinsame Telegramm der Vorsitzenden der Fuldaer und der Freisinger Bischofskonferenz, der Kardinäle Felix von Hartmann und Franz von Bettinger an Kaiser Wilhelm II., in dem beide im Namen des gesamten deutschen Episkopats „schmerzliche Entrüstung“ über die antideutschen Angriffe aus den Rei-

41 Arnold J. Rosenberg (Hrsg.): Der deutsche Krieg und der Katholizismus. Deutsche Abwehr französischer Angriffe. Paderborn 1915. In weiteren Variationen erschienen unter Beteiligung Rosenbergs im Laufe des Jahres entsprechende Publikationen.

42 Georg Pfeilschifter (Hrsg.): Deutsche Kultur, Katholizismus und Weltkrieg. Eine Abwehr des Buches „La Guerre allemande el le Catholicisms“, Freiburg i.Br. 1915. Darin u.a. Michael von Faulhaber: Unsere religiöse Kultur, S. 451-476.

43 Michael Faulhaber (1869-1952) leitete von 1911 bis 1917 das Bistum Speyer und wurde 1913 vom bayerischen König Ludwig III. in den persönlichen Adelsstand erhoben. Als Nachfolger des verstorbenen Kardinals Franz von Bettinger wurde er 1917 zum Erzbischof von München ernannt und 1921 von Benedikt XV. zum Kardinal erhoben.

44 Arnold J. Rosenberg: Der deutsche Krieg und der Katholizismus. Entgegnungen auf das Buch „La Guerre allemande et le Catholicisme“. In: Theologie und Glaube, 7. Jg. 1915, Heft 5 (Mai), S. 353-369.

45 Kölnische Volkszeitung, Nr. 487 v. 17.6.1915 Hervorhebungen im Original. 
hen der französischen Kirche bekundeten und zusagten, in dieser Angelegenheit im Vatikan Beschwerde zu führen. 46

Bereits Anfang Juni 1915 hatten deutsche Zeitungen die überfällige nationale Gegenwehr auf diesen "Gipfel bisheriger Lügenfeldzüge“ des Auslands gegen das Deutsche Reich begrüßt. Dass an den französischen Veröffentlichungen auch Bischöfe mitgewirkt hatten, wurde als Indiz dafür interpretiert, dass der westliche Nachbar den militärischen Konflikt in einen Religionskrieg umwandeln wolle. Da aber aus Sicht der deutschen Bischöfe vor allem die Aktivitäten Rosenbergs eine rein „politische Tendenz" aufwiesen, beteiligten sie sich daran nicht. Die Berichterstattung der Zeitungen sowie das Telegramm der beiden Kardinäle an den Kaiser empfand der Autorenkreis um den Paderborner Theologen gleichwohl als Rückenstärkung. Als Rosenbergs Antwortschrift erschien, standen auf der Unterzeichnerliste nicht weniger als 126 Persönlichkeiten des katholischen Lebens in Deutschland. ${ }^{47}$

Mit „Frankreichs Versündigungen am Katholizismus" setzte sich Mitte Juli in der Münchner "Allgemeinen Rundschau“ der katholische Reichstagsabgeordnete Eugen Jaeger auseinander. Durchaus üblich war es, dass die Katholische Presse und kirchliche Zeitungen punktuell oder auch regelmäßig Politiker und Militärs ohne sonderliche Unterscheidung von ihrem sonstigen redaktionellen Stab als Autoren heranzogen. Jaeger sprach den Franzosen die Eignung ab, sich als „Vertreter des Katholizismus aufzuspielen", da ihr Staat im Bunde mit den kirchenfeindlichen "Freimaurern" und dem orthodoxen „schismatischen Russland" als seinen "Lieblingsbundesgenossen “gegen Papst, Kirche und auch gegen Deutschland stehe. ${ }^{48} \mathrm{Im}$ historisch-politischen Rückblick verstieg er sich gar zu der eigenwilligen Interpretation, Frankreich habe seinerzeit aus Machtstreben der Reformation Martin Luthers zum Durchbruch verholfen, um nicht nur den Konkurrenten Deutschland zu schwächen, sondern letztlich auch das Papsttum zu zerstören. Die Geschichte indes beweise anderes: Im „katholischen“ Frankreich, triumphierte Jaeger, spiele die Kirche keine Rolle mehr, während in dem dort herablassend betrachteten „protestantischen" Deutschland gegenwärtig ein reiches religiöses Leben und sittlicher Ernst herrschten.

Auszüge aus der bereits bekannt gewordenen Antwort Benedikt XV. auf die Eingabe der deutschen Bischöfe veröffentlichte Ende Oktober

46 Vgl. Kölnische Volkszeitung, Nr. 497 v. 21.6.1915. Hier war das Telegramm der Aufmacher.

47 Vgl. Kölnische Volkszeitung, Nr. 552 v. 9.7.1915.

48 Allgemeine Rundschau, 12. Jg. Nr. 29 v. 17.7.1915. Hervorhebungen im Blatt. 
1915 die „Germania“, eher auf Mäßigung bedacht als das Kölner Schwesterblatt. Die hauptstädtische Zeitung hob das Bemühen des Papstes hervor, dem national-militärischen Gegeneinander zumindest die religiös-kirchliche Dimension zu nehmen. Vom Weg des Friedens, so die „Germania“, würden „weit abirren, die etwa glauben sollten, es sei ihnen erlaubt, die Handlungen der Katholiken eines anderen Volkes durch Wort und Schrift in einer Weise herabzusetzen [...] und so neuen Zunder zu der Erbitterung zu liefern, deren Glut sie durch Gerechtigkeit des Urteils und Milde der Gesinnung löschen sollten". ${ }^{49}$ War die Mahnung Benedikt XV. auch an alle Kontrahenten adressiert, so las doch jede Seite daraus vor allem die Bestätigung für das jeweils eigene Vorgehen heraus. Eines der Beispiele dafür, dass es Papst und Heiligem Stuhl praktisch unmöglich war, sich einer parteiischen und nationalen Vereinnahmung zwischen den Fronten zu entziehen.

Die erhoffte Mäßigung blieb aus. Ende 1915 legte die französische Seite erneut nach. Wiederum unter Leitung von Alfred Baudrillart gab das "Comité Catholique de Propaganda Francaise a L'Etranger" mit Druckerlaubnis des Pariser Kardinals Léon-Adolphe Amette vom 2. Dezember 1915 die Schrift „L'Allemagne et les Alliés devant la Conscience chrétienne" (Deutschland und die Alliierten vor dem christlichen Gewissen) heraus. ${ }^{50}$ Auch dieser Band beinhaltete Einzelaufsätze von Bischöfen und Gelehrten. Welche Mittel dabei als geeignet erschienen, belegt etwa die einleitende Abhandlung, in der Baudrillart an ein mehrere Jahre zurückliegendes Gespräch zwischen dem nunmehrigen Kardinal Louis Lucon von Reims ${ }^{51}$ und Papst Leo XIII. (1878-1903) in Rom erinnert, in dem letzterer sich sehr abfällig über Deutschland und den dort herrschenden „Geist Luthers“ geäußert habe.

Besonderes Augenmerk der katholischen Presse galt der einleitenden Abhandlung „Frankreich und Deutschland vor der christlichen Lehre" in der neuen französischen Anklageschrift, stammte sie doch aus der Feder eines Bischofs - Henri Luis Chapon von Nice ${ }^{52}$. Einerseits zeigten sich

49 Germania, Nr. 501 v. 29.10 .1915$.

50 Alfred Baudrillart (Hrgs.): L'Allemagne et les Allies devant la Consciense chrétienne. Paris 1915. Und wiederum erschien gleichzeitig auch ein neuer bebilderter Dokumentationsband zu deutschen Kriegsgräuel: „La Guerre allemande et le Catholicisme, Album N $\mathrm{N}^{\circ}$ 2. 32 Seiten, Querformat. Paris 1915.

51 Louis-Henri-Joseph Lucon (1842-1930) war seit 1887 Bischof von Belle und führte das von Baudrillart erwähnte Gespräch in Rom 1888. Im Jahr 1906 wurde er von Pius X. zum Erzbischof von Reims ernannt und 1907 zum Kardinal erhoben.

52 Henri Luis Chapon, geboren am 14. März 1845, wurde laut kirchlicher Quellen als 51-Jähriger 1896 Bischof im südfranzösischen Nice (Nizza). Das Ende seiner 
die deutschen Rezensenten erschrocken über den Ton des Bischofs. Andererseits erschien ihnen Chapon als willkommenes Beispiel eines feindlich gesonnenen und verblendeten Bischofs. Dieser sehe in den Deutschen ein „Barbarenvolk“, hinter dessen Taten und Verbrechen ein heidnischer Pangermanismus stehe, der "alles heiligt, eingibt und leitet". Völlig unzulässig unterstelle Chapon dem Deutschen Reich, von einem Herrschaftsanspruch über alle anderen Völker getrieben zu sein. An die Stelle des wahren Gottes, wurde Chapon zitiert, sei ein Götzendienst, ein Glaube an den „alten deutschen Gott“, an den „germanisierten Moloch, das Symbol des deutschen Egoismus", getreten. ${ }^{53}$ Einer der wenigen Publizisten, die angesichts dieser Bedrohung der innerkatholischen Verständigung doch einen Brückenschlag suchte, war Matthias Reichmann SJ. In der Jesuiten-Zeitschrift „Stimmen aus Maria Laach“ hatte er bereits im Frühjahr französische Meinungen zur aktuellen Fehde zusammengestellt, die belegten, dass es beim westlichen Nachbarn durchaus auch Katholiken und Periodika gab, die den nationalistisch-polemischen Überschwang der „La Guerre“-Autoren nicht teilten. 54

\section{Der Beginn des Gaskriegs}

Die Schlagzeile „Die Verwendung von Gasgeschossen“ in der Mittagsausgabe der „Kölnischen Volkszeitung“ am 30. Juni 1915 versprach auf den ersten Blick mehr, als der dazugehörige Artikel hielt. Dem vergleichsweise kurzen Beitrag waren außer der Bestätigung des Faktums keinerlei Erklärungen oder Details zum Giftgaseinsatz durch deutsche Truppen zu entnehmen. Der "Aufmacher“ in dieser Ausgabe des Kölner Zentrums-Blattes bezog sich zudem auf ein Ereignis an der Westfront in Belgien, das bereits fast zwei Monate zurücklag.

Am 22. April 1915 hatten die deutschen Truppen erstmals bei Ypern in Westflandern Giftgas gegen die Stellungen der Franzosen eingesetzt. Jener Tag im April vor 90 Jahren markiert den Beginn einer neuartigen Kriegsführung - der mit chemischen Kampfstoffen. Um Bewegung in den

Amtszeit weisen die Quellen nicht aus. Als nächster Bischof von Nice wird Paul Remond (1873-1963) aufgeführt, der hier ab 1930 bis zu seinem Tod als Bischof wirkte.

53 Vgl. Henri Luis Chapon: La France et l'Allemagne devant la doctrine chrétienne. In: Baudrillart (Hrs.): L'Allemagne et les Allies devant la Conscience chrétienne, Paris 1915.

54 Matthias" Reichmann SJ: „Umschau“. In: Stimmen aus Maria Laach“, 88 Jg, Heft 5, S. $488-491$. 
Stellungskrieg im Westen zu bringen, hatte der deutsche Generalstab erstmals - und völkerrechtswidrig - Giftgasgeschosse gegen die feindlichen Stellungen zum Einsatz gebracht und damit eine neue Spirale der allseitigen Aufrüstung ausgelöst. Historiker und Experten schätzen heute, dass allein der erste deutsche Einsatz von Giftgas an diesem 22. April 1915 bei Ypern unter den französischen Soldaten bis zu 1200 Tote und 3000 Verletzte forderte. ${ }^{55}$

Der deutsche Giftgaseinsatz war auf Grund des „Burgfriedens“, der militärischen Zensur und ebenso der journalistischen Selbstzensur für die deutsche Presse kein Anlass zu kritischen Anfragen. Die „Kölnische Volkszeitung" zeigt zu dieser Zeit exemplarisch, dass auch die Zentrumsund die Kirchenpresse diesen qualitativen Sprung in der Kriegsführung und damit verbundene ethische Fragestellungen nicht problematisierte. Sie enthielt sich jeder eigenen Meinung, veröffentlichte den Beitrag allerdings schon in rechtfertigender Absicht auf der Titelseite. Im Text selbst wurde eine zuvor publizierte Erklärung der regierungsamtlichen „Norddeutschen Allgemeinen Zeitung“ wiedergegeben, in der diese französische und britische Kritik am deutschen Giftgaseinsatz als „Fälschungen“ und durch „Bestechungsversuche" herbeigeführt zurückgewiesen hatte. ${ }^{56}$ Die exponierte Stellung dieses Dementis in der „Kölnischen Volkszeitung“ kann immerhin als Indiz angesehen werden, wie ernst doch die Reaktionen im Ausland auf den Verstoß Deutschlands gegen die Haager Landkriegsordnung hinter den Kulissen genommen wurden.

\section{Friedensinitiativen und Siegeshoffnungen bis 1918}

Die im Verlauf des Jahres 1915 vor allem an den Beispielen der „Kölnischen Volkszeitung“ und der „Germania“ erkennbar gewordenen Antagonismen zwischen den Anhängern eines „Siegfriedens“ und eines

55 Rolf-Dieter Müller: „Gaskrieg“. In: EEW, S. 519-522, hier S. 520. Der deutsche Gaseinsatz ging auf den unmittelbaren Vorschlag des durch und durch patriotischen und militaristischen Chemikers Fritz Haber (1868-1934) zurück, der 1893 vom Judentum zum Protestantismus konvertiert war. Habers Frau, ebenfalls promovierte Chemikerin, beging wenige Tage, nachdem ihr Mann den Giftgaseinsatz bei Ypern selbst überwacht hatte, aus Protest gegen sein Engagement Selbstmord. - In vielen Staaten wurde bereits an der Entwicklung von Kampfgasen gearbeitet, worauf sich auch Huber berief, als er der OHL seine Entwicklungen empfahl. Alle Kriegsparteien setzten im Ersten Weltkrieg rund 112000 Tonnen Gas ein, davon Deutschland schätzungsweise 52000 Tonnen. Der Einsatz blieb auf den Frontbereich beschränkt. Vgl. Rolf-Dieter Müller: a.a.0. und Dieter Storz: „Haber, Fritz“. In: EEW, Sp. 540.

56 Kölnische Volkszeitung, Nr. 524 vom 30. Juni 1915. 
„Verständigungsfriedens“ in der katholischen und teilweise auch in der kirchlichen Presse hat sich in den folgenden Kriegsjahren verstärkt. Dabei blieben allerdings die Befürworter einer Verständigungslösung in der Minderheit.

Besonders deutlich zeigten sich die gegensätzlichen Standpunkte in der Beurteilung der vom Zentrums-Abgeordneten Matthias Erzberger initiierten Reichstagsresolution für einen Verständigungsfrieden vom 19. Juli 1917. Die kontroversen Debatten wiederholten sich, als dann Papst Benedikt XV., obwohl ein Scheitern seiner Initiative bereits absehbar war, zum dritten Jahrestag des Kriegsausbruchs, am 1. August 1917, seine Note für einen Verständigungsfrieden veröffentlichte. In beiden Fällen formulierte die Berliner „Germania“ lobend, während sich die "Kölnische Volkszeitung“ als Sprachrohr jener exponierte, die auf beide Friedensvorstöße enttäuscht und zurückhaltend reagierten. Mit den Begriffen „Scheidemannfrieden“ oder „Hindenburgfrieden“ wurden die als unüberbrückbar dargestellten inhaltlichen Pole „Verzichtfriede“ oder „Siegfriede“ in der Diskussion personalisiert.

Die Niederlage im November $1918 \mathrm{kam}$, so erscheint es zumindest weithin in der veröffentlichten Meinung, nach den Jahren der Propaganda und geschürten Hoffnungen geradezu unerwartet und abrupt. Erst recht wurde in diesem Klima, in dem der "Krieg in den Köpfen“ 57 bis in die Weimarer Republik hinein weiterging, der Friedensvertrag von Versailles, in dem die Siegermächte ihre Ansprïche diktierten und Deutschland die alleinige Schuld am Kriegsausbruch anlasteten, als „Schmachfriede" empfunden. Weitsichtig hat vor den Folgen dieses Vertragswerkes auch Papst Benedikt XV. gewarnt.

57 Vgl. Wolfgang J. Mommsen: a.a.0., S. 200-210. Gerd Krumreich: Versailles 1919. Der Krieg in den Köpfen. Essen 2001. 